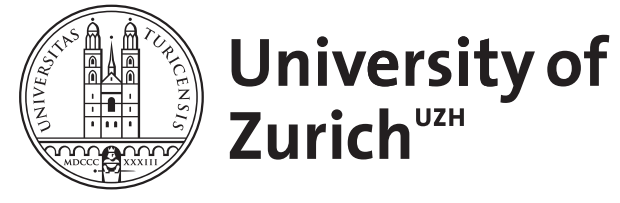

\title{
Aufbruch ohne Zerstörung
}

\author{
Abbt, Christine
}

DOI: https://doi.org/10.1515/dzph-2017-0052

Posted at the Zurich Open Repository and Archive, University of Zurich ZORA URL: https://doi.org/10.5167/uzh-139736

Journal Article

Published Version

Originally published at:

Abbt, Christine (2017). Aufbruch ohne Zerstörung. Deutsche Zeitschrift für Philosophie, 65(4):781-785. DOI: https://doi.org/10.1515/dzph-2017-0052 


\section{Buchkritik}

\section{Christine Abbt* \\ Aufbruch ohne Zerstörung}

DOI 10.1515/dzph-2017-0052

Dieter Thomä. Puer robustus. Eine Philosophie des Störenfrieds. Berlin, Suhrkamp, 2016, 715 S.

Noch bevor Familienbeziehungen ein anerkanntes Thema der Praktischen Philosophie darstellten, setzte sich Dieter Thomä philosophisch mit dem Vater und dessen Rolle auseinander. Sein neues Buch nun ist dem Kind gewidmet, genauer dem robusten Knaben. Von Thomas Hobbes 1647 im Vorwort der zweiten Auflage von De cive ins Leben gerufen und als gefürchteter Störenfried entworfen, inspirierte der kleine Kerl die Philosophen in der Folge während dreier Jahrhunderte. Dann allerdings geriet er in Vergessenheit. Mit Verve und Sympathie rückt Thomä mit seiner umfangreichen Untersuchung das kräftige Kind jetzt wieder in vielen seiner Facetten in die philosophische Mitte. Von Kapitel zu Kapitel lässt er die bisher weitgehend unbeachtete Figur in unterschiedlicher Gestalt auftreten, stellt einmal das Böse und Schlechte, das Gefürchtete daran vor, ein anderes Mal das Rettende, Freie, Labende, und meistens von beiden Anteilen etwas. Von allen Varianten interessiert den Verfasser der eindrücklichen Studie dabei ganz besonders jener Störenfried, durch den etwas Starres aufbricht und in Bewegung kommt. Im Zentrum jedenfalls der normativen Aufmerksamkeit von Thomä steht jener „Gelegenheitstäter“, der sich seiner eigenen Schwellensituation bewusst bleibt und sein Störungspotential mit konstruktivem „Überschuss“ verbindet. Es handelt sich dabei um Formen von Störung ohne grundlegende oder totale Zerstörung, um jene Möglichkeiten des Aufbegehrens gegen Konformismus, die nicht einzig die Masse oder das eigene Ego ins Recht setzen, sondern den kritischen Sinn kräftigen. Der puer robustus trägt, so macht die Untersuchung an vielen Beispielen kenntlich, nicht immer das Gesicht dieses ,exzentrischen“ Störenfrieds. Das empathische philosophische und politische Interesse des Verfassers gilt allerdings ihm.

Eine Mischung aus Abenteuergeschichte und Abhandlung wolle er vorlegen, meldet sich in der Einleitung der Verfasser selbst kommentierend zu Wort. Das Buch ist mehr. Es lädt mit seiner klaren, direkten, auch bildkräftigen Sprache tat-

*Kontakt: Christine Abbt, christine.abbt@unilu.ch 
sächlich zu abenteuerlichen Ausflügen und unerwarteten Begegnungen ein und bietet gleichzeitig eine gelungene Einführung in die Politische Philosophie von Hobbes bis Schmitt oder Strauss, bei der häufig fast vergessen geht, wie viel man lernt, weil es so gut und spannend verfasst und zu lesen ist. Darüber hinaus ziehen sich systematische Fragen wie ein roter Faden durch das gesamte, im Haupttext fast sechshundert Seiten lange Buch, die sich an der Figur des starken Jungen immer wieder neu kristallisieren und diskutieren lassen. Die Fragen richten sich auf das Werden und Aufblühen, auf die Übergänge und Entwicklungen von Menschen, Ordnungen, Ideen und Taten. Bereits in seiner Schöpfungsgeschichte bei Hobbes ist das Augenmerk ausgehend vom puer robustus auf jene Transitionen und Kippmomente zwischen Kind und Erwachsenem, zwischen Tat und Untat, zwischen Heldentum und Verbrechen gerichtet. Thomä setzt ebenda noch einmal ein und umkreist ebenso virtuos wie konzis die rätselhaften Veränderungen, die aus einem Menschen einen Anderen werden lassen bzw. die Deutungen und Zuschreibungen dazu. Bei Hobbes etwa, dem Vater des puer robustus, wird das Ausbleiben von Veränderung zum Problem. Der Knabe reift nicht zum Mann und auf diese Weise, als nicht erwachsen gewordenes Kind, gilt er als Bedrohung der Ordnung von Leviathan. Die Aussage von Hobbes mit der von Thomä identifizierten Initialzündung lautet: „A wicked man is almost the same thing with a childe growne strong and sturdy, or a man of a childish disposition.“

Der Auftakt des Buches zum Reigen der Störenfriede und Quertreiber der Philosophiegeschichte von der Neuzeit bis nach China im Jahr 1957 ist fulminant. Nicht nur ist die Kritik an Hobbes' Menschenbild und Staatsvorstellung treffend, es werden in diesem Kapitel auch Figuren aufs Tapet gebracht, die sonst mit Bezug auf Hobbes nur selten zur Diskussion kommen. Eine Fülle von Egozentrikern wie etwa den Narren, den Tollwütigen oder den Epileptiker führt Thomä vor und legt damit dar, dass die Vielfalt der menschlichen Varietäten und Handlungsmotive, wie sie bei Hobbes selbst vorkommen, nicht allesamt plausibel unter die Maxime der Selbsterhaltung zu subsumieren sind. Die egozentrischen Rebellen sind aber nicht die einzigen, die ausscheren. Bestechend ist Thomäs Analyse der sogenannten Angepassten im Inneren der Ordnung, die ihrerseits das Potential zum Störenfried besitzen. Als Vertragsstiftende sind die Angepassten „authors“. Bei Hobbes dürfen sie hingegen nicht und nie zu „actors“ werden. Die Handlungsmacht obliegt einzig und allein dem Souverän, dem Leviathan. Diese strikte Trennung zwischen „author“ und „actor“, so führt Thomä aus, ist höchst problematisch und auf Dauer nicht als gewünschter Friedenszustand zu denken. In der radikalen Ablehnung gegenüber der Schauspielerei und dem Theater zeigt sich, dass Hobbes sich dieser Problematik durchaus bewusst war. Der Schauspieler gehört nicht nur zur Gruppe der „Herrenlosen“, er steht in Reinform für diese Lebensform, so Thomä. Genau dies lässt andere Philosophen aufmerken. Diderot 
etwa wird daran seine eigene, positive Perspektive des puer robustus entwickeln. Auch Rousseau lässt Hobbes nicht gewähren und verpasst dem robusten Jungen das Konterfei des guten Wilden.

Von Hobbes über Rousseau zu Diderot und weiter zu Friedrich Schiller, Victor Hugo und Richard Wagner! Es sind hier bekannte Namen, mit denen die Lesenden durch das Vermittelte auf neue Weise ins Gespräch treten können. Dabei werden enge Bezüge zwischen Denkern sichtbar, die bei anderer Interessenlage wenig Beachtung finden. Interessant etwa ist der aufgedeckte Zusammenhang zwischen Tocqueville, Mill und Nietzsche und deren Verweise auf China als Beispiel für Konformismus und Stillstand. Thomä verknüpft die Ansichten der drei Denker über demokratische Ordnung und Regelbruch gekonnt und vertieft ihre Differenzen immer auf die Frage bezogen, welche Art von Aufbegehren, Hervorragen, Ausscheren als demokratisch wünschenswert oder aber problematisch zu gelten hat. Dass das Denken der drei Philosophen jeweils auch im Verlauf des eigenen Lebens Entwicklungen zeitigt, wird dabei nicht unterschlagen. Im Gegenteil bietet dieser biographisch-philosophische Überblick seinerseits Hinweise darauf, wie sich Überlegungen beeinflussen und verändern und inwiefern Prozesse des Denkens Prozesse des Schaffens umfassen.

Der Bogen von der Neuzeit in die Mitte des 20. Jahrhunderts ist faszinierend prall gespannt und das Bild des politischen Störenfrieds, der ohne böse Absicht aufbricht, der Grenzen auslotet, ohne den radikalen Umbruch einzuleiten, und der damit für die Demokratie lebenswichtig ist, gewinnt von Seite zu Seite markantere Konturen. Dies alles ist hochspannend und wichtig. Es ist Demokratietheorie im allerbesten Sinn! Darauf folgt im Buch bedauerlicherweise ein Bruch, einerseits historisch - einige Jahrzehnte erscheinen nur am Rande oder gar nicht -, andererseits stilistisch - das letzte Kapitel des Buches gleicht einer Reflexion verschiedener Aspekte der Gegenwart, in die sich die Stimme des Verfassers in unterschiedlichen Tonlagen einmischt, einmal explizit Stellung bezieht, einmal andere zu Wort und miteinander ins Gespräch kommen lässt. Zwischen 1957 und 1989 taucht der puer robustus auf der philosophischen Bühne offenbar nur noch marginalisiert auf? In der Zeit der Bürgerrechtsbewegungen, der Zeit des Feminismus, der Zeit der intensiven kritischen Aufarbeitung der Shoah, in jener Zeit, in der sich ein neues Verständnis der Geschlechterordnung und Sexualität durchsetzte, in der Zeit, in der die Herausbildung einer Sprache, die den Anforderungen politischer Korrektheit entspricht, noch nicht $\mathrm{zu}$ Konformismus tendierte, sondern zu Aufbruch, Erneuerung, zu mehr Freiheit, Gleichberechtigung und Selbstbestimmung für alle, tritt der kräftige Knabe in den Hintergrund? Und ab 1989 erfahren die Störenfriede im Westen eine Gesellschaft, welche die Ausnahme zur Regel erhebt? 
Geleitet von einer heute beinahe vergessenen, so produktiv herausfordernden Denk-Figur wie der des puer robustus handelt die Untersuchung von Thomä von schreibenden und denkenden Männern, die kleine und große Wilde entwerfen und damit andere schreibende, denkende Männer bis in die Gegenwart inspirieren. Sie handelt von theoretischen und praktischen Helden, von Dissidenten und Unterdrückern. Dabei können wohlbekannte Männer der Weltgeschichte wie etwa Marx oder Horkheimer neu kennengelernt werden, aber auch vermutlich weniger bekannte wie insbesondere Tan Tianrong, ein Wortführer im Rahmen der Hundert-Blumen-Gesellschaft, die sich 1957 an der Universität Peking konstituiert, und der, angeregt durch Mao selbst, die „Diktatur des Proletariats“ feiert und gleichzeitig vermeintlich großzügig zur freien Meinungsbildung aufruft, den Protest wagt. Vierhunderttausend Menschen werden in der Folge verurteilt oder verbannt. Im Werk von Thomä ist der Protest-Text von Tan Tianrong zum ersten Mal in Übersetzung in ganzer Länge nachzulesen. Die Überlegungen zur Bedeutung der Hundert-Blumen-Bewegung, die Würdigung Tans und seines Verständnisses von Aufbruch und Evolution, die Darlegung der Zusammenhänge zwischen dieser Bewegung und der Kulturrevolution der Jahre nach 1966 bis in die Gegenwart zum Künstler Ai Weiwei bilden einen Glanzpunkt innerhalb der Untersuchung. Thomä verknüpft gekonnt verschiedenes, teilweise selten diskutiertes Material und bleibt dabei stets bei der so relevanten Frage, wie das wohlwollende Kraftvolle, das auf Vermehrung von Freiheit für alle drängt, im Kontext von Machtmonopolisierung und Ordnungen $\mathrm{zu}$ beschreiben, $\mathrm{zu}$ verstehen und $\mathrm{zu}$ begründen ist.

Seltener sind die Begegnungen mit Frauen der Weltgeschichte. Hannah Arendt oder Judith Butler und manche andere Philosophin tauchen zwar auf, sie bleiben aber überraschend wenig vertieft. Ist die Störung der Ordnung, ohne die Ordnung zu zerstören, vor allem männlich besetzt? Waren es nicht oft Frauen, die die Formen von Anpassung und Ermächtigung meisterhaft angewandt, reflektiert und verfeinert haben? Die Wichtigkeit der „Arme der Mutter“ streichen Tocqueville und mit ihm auch Thomä hervor. Über weite Strecken der Studie bleiben die Aufmüpfigen, die Heldinnen und Kämpferinnen mit und ohne Kinder und auch all die ambigen Personen und Figuren, die ihrerseits die Kategorien von weiblich und männlich grundlegend in Frage stell(t)en, außen vor. Hans Kelsen, so zeigt Thomä auf und setzt mit diesen Ausführungen innerhalb des Buches einen weiteren Glanzpunkt, war erst nötig, damit der puer robustus auch seine weibliche Seite zu entdecken vermochte. Die von Kelsen reflektierte und vorgeschlagene Anerkennung der bisexuellen Anlage des Menschen als Bedingung für ein neues Verständnis der Gleichheit aller Menschen ist allerdings bis heute nicht realisiert. Dem Anliegen wird auch von Thomä selbst nicht vollumfänglich Rechnung getragen. 
Die puella robusta tritt zwar auf, etwa in der Gestalt der Esmeralda in Victor Hugos Roman Der Glöckner von Notre-Dame oder der Antigone von Sophokles. Eine vertiefte Ausarbeitung dieser Figuren fehlt allerdings in der Untersuchung. Genderspezifisch interessante Spielarten werden nicht eingehend in den Blick gerückt und das auch dort nicht, wo es sich anbieten würde - denn diese Figuren gibt es: Von Xanthippe, die ihren Mann nicht verstehen will, die Liebe über das Pathos des Asketen stellt und damit viel Häme in Texten der Philosophiegeschichte einstecken musste, bis hin zu noch weit besser in die Reihe der puellae passenden Figuren der Literatur gäbe es doch manche bemerkenswerte zu wählen. Mignon von Goethe etwa oder Penthesilea von Kleist wären da zu nennen, oder das Mischwesen Undine von De la Motte Fouqué, die dann bei Ingeborg Bachmann hundert Jahre später noch einmal antritt, klagt und als Störung der unerbittlichen Ordnung der oberflächlichen Sprache auf- und abtritt. Auch bei Rousseau wäre noch einmal zu fragen, was Menschen zu Helden oder Heldinnen macht. Sophie, die puella robusta in Émile, muss gebändigt werden. Ihre natürliche kindliche Neugier ist abzutöten, weil sie ein Mädchen ist und eben darum nicht robust werden oder bleiben soll. Thomä erwähnt, dass die Frauen, wie Rousseau sie sehe und kritisiere, „eine erstaunliche Ähnlichkeit mit dem puer robustus“ aufweisen (101), und dass die Abweisung der Passion mit der Ausgrenzung der Frau bei Rousseau einhergehe, dass also die von Rousseau versprochene Gleichheit Ungleichheit evoziere. Dabei belässt er es.

Als ob der Verfasser der Philosophie des Störenfrieds dieses Brachland selbst bemerkt hätte, überlässt er ganz am Ende noch Henrik Ibsen das Wort, einem Autor, der neben anderen auch geschlechterbezogene Zwänge besonders scharf und exakt in den Blick zu rücken wusste. Nora, eine Figur aus dem Drama Ein Puppenheim, knallt darin die Türe zu. Sie will sich nicht weiter zurückbinden lassen. Sie will sich selbst ein Bild der Gesellschaft machen und wehrt sich dagegen, dass ihre Neugier als Fieber, ihr Aufbegehren als Krankheit abgestempelt und neutralisiert wird. Nora geht und schlägt die Türe zu. Das Geräusch, das beim Schlagen einer Türe verursacht wird, so Thomä, sei beim Öffnen wie beim Schließen dasselbe. Es wäre Ibsens Nora und der Philosophie zu gönnen, dass die Türe auch diesbezüglich nicht zu-, sondern aufgeschlagen wird. Spannend wäre es, wenn der Autor, der über Väter und Söhne, über kräftige Knaben und selbstbewusste Störenfriede so interessant, gehaltvoll und grundlegend berichtet, eines seiner nächsten Bücher der Denk-Figur einer mutigen Frau oder einem bisexuellen Mischwesen widmete. Das wäre eigens immer noch ein philosophischer Stör- und auf jeden Fall ein weiterer philosophischer Glücksfall! 